\title{
Saturación de oxígeno, respiración periódica y apnea durante el sueño en lactantes de 1 a 4 meses a 2560 metros de altura
}

\author{
Oxygen saturation, periodic breathing and apnea during sleep in infants \\ 1 to 4 month old living at 2,560 meters above sea level
}

\author{
Dr. Santiago Ucrósa , Dra. Claudia Granados, M. Sc. , Dra. Karem Parejoc, Dr. Fernando Guillén ${ }^{d}$, \\ Dr. Fausto Ortega'e, Dra. Sonia Restrepof, Fabián Gil, M. Sc. ${ }^{g}$ y Estud. Miriam Guillén ${ }^{h}$
}

\begin{abstract}
RESUMEN
Los estudios sobre polisomnografía en lactantes entre los $2200 \mathrm{~m}$ y los $2800 \mathrm{~m}$ de altura son escasos. Esta investigación tuvo como objetivo describir la saturación de oxígeno $\left(\mathrm{SpO}_{2}\right)$ durante el sueño en niños de 1-4 meses a $2560 \mathrm{~m}$ y, como objetivos secundarios, la descripción de los índices de apnea y respiración periódica (RP). Se realizaron polisomnografías en 35 lactantes sanos de 1-4 meses en Cuenca (Ecuador) a $2560 \mathrm{~m}$. La mediana para la $\mathrm{SpO}_{2}$ fue $92 \%$ y $4,9 \%$ para la RP. El índice de apnea central tuvo una mediana de 23,7/hora, que disminuyó a 4,5/hora al descontar las apneas asociadas a
\end{abstract}

a. Departamento de Pediatría. Fundación Santa Fe de Bogotá.

b. Departamentos de Pediatría y Epidemiología Clínica y Bioestadística. Pontificia Universidad Javeriana.

c. Departamento de Neurología y Laboratorio de Sueño. Fundación Clínica Shaio. Bogotá, Colombia.

d. Departamento de Pediatría. Hospital del Río. Universidad del Azuay. Cuenca, Ecuador.

e. Departamento de Pediatría. Hospital Luis Fernando Martínez. Cañar, Ecuador.

f. Departamento de Pediatría. Hospital de La Misericordia y Fundación Santa Fe de Bogotá, Universidad de los Andes.

g. Departamento de Epidemiología Clínica y Bioestadística. Pontificia Universidad Javeriana.

Bogotá, Colombia.

h. Estudiante de Medicina. Universidad del Azuay. Cuenca, Ecuador.

Correspondencia:

Dr. Santiago Ucrós: santiago_ucros@yahoo.com

Financiamiento: Esta investigación fue parcialmente financiada con aportes de la Fundación Conocimiento, Bogotá, Colombia (Grant PI-FC/001-2012), el Fondo de Educación del Departamento de Pediatría de la Fundación Santa Fe de Bogotá y el Fondo para Investigación de la Universidad del Azuay, Cuenca, Ecuador.

Conflicto de intereses: Ninguno que declarar

Recibido: 14-2-2015

Aceptado: 23-4-2015
RP. No hubo correlación entre la $\mathrm{RP}$ y la $\mathrm{SpO}_{2}$. Conclusión: la $\mathrm{SpO}_{2}$ fue inferior a los valores del nivel del mar, y la RP y el índice de apnea central, mayores. Cuando las apneas asociadas a RP se descontaron, el índice de apnea central fue similar al del nivel del mar.

Palabras clave: sueño, lactante, altitud, oxígeno, polisomnografía.

http:/ /dx.doi.org/10.5546/aap.2015.341

\section{INTRODUCCIÓN}

Desde su introducción en 1975, la oximetría de pulso se usa rutinariamente y se considera un nuevo signo vital. La saturación de oxígeno de la hemoglobina $\left(\mathrm{SpO}_{2}\right)$ en niños, en sus primeros meses de vida, ha sido establecida durante la vigilia a diferentes alturas, pero los datos para niños dormidos son más escasos. Estudios durante el sueño se han hecho en Colombia, ${ }^{1,2}$ Bolivia, ${ }^{3}$ Perú, ${ }^{4}$ China, ${ }^{5}$ Estados Unidos ${ }^{6} \mathrm{y}$ Argentina. ${ }^{7}$

Esta investigación tuvo como objetivo principal la descripción de la $\mathrm{SpO}_{2}$ durante el sueño en niños de 1-4 meses a $2560 \mathrm{~m}$ de altura. Como objetivos secundarios, estuvieron la descripción de los índices de apnea y de la respiración periódica $(\mathrm{RP})$.

\section{METODOLOGÍA}

El estudio fue realizado en Cuenca, Ecuador, a $2560 \mathrm{~m}$ de altura. El protocolo fue aprobado por los comités de ética de las instituciones participantes. El tamaño de muestra se calculó utilizando el programa TAMAMU $1.1 .^{8}$ Las estimaciones fueron hechas asumiendo un error tipo I de 0,05 con una desviación estándar de 3,4 para el promedio de la $\mathrm{SpO}_{2}$ (basándose en el estudio de la Universidad del Bosque en Bogotá), ${ }^{1}$ con un nivel de confianza del $95 \%$ y una precisión absoluta del $2 \%$. Se hizo un análisis descriptivo usando medianas y percentiles y teniendo en 
cuenta la distribución asimétrica de los datos. Las medianas fueron comparadas por grupos de edad para la $\mathrm{SpO}_{2}$, la $\mathrm{RP}$ y el índice de apnea central (IAC), por medio del test de Kruskal-Wallis. Los valores de $\mathrm{p}<0,05$ fueron considerados estadísticamente significativos.

Los lactantes incluidos debían tener historia de un embarazo a término, peso al nacer de $2500 \mathrm{~g}$ o mayor y ausencia de patología perinatal o actual. Todos los participantes del estudio eran nativos y habían vivido siempre en Cuenca, al igual que sus padres.

Los padres que aceptaron participar firmaron un consentimiento informado. Las polisomnografías fueron realizadas en una habitación del hospital, y los niños estuvieron acompañados por sus madres. Para su realización, se utilizó un equipo BWII aprobado por la Administración de Medicamentos y Alimentos (Food and Drug Administration; FDA, por sus siglas en inglés) y que cumplía con los requisitos de la Academia Americana de Medicina del Sueño (AASM). La $\mathrm{SpO}_{2}$ se midió con un sensor de oxímetro Nonin modelo 8008J. Las polisomnografías debían tener un mínimo de 180 minutos de tiempo total de sueño para ser tenidas en cuenta. La interpretación se hizo de acuerdo con las recomendaciones de la AASM. ${ }^{9}$ Se utilizó la siguiente definición de RP estipulada por la ASMM: "Se califica como RP un evento caracterizado por 3 o más apneas centrales, de más de 3 segundos de duración, separadas por 20 o menos segundos de respiración normal. Las apneas centrales que ocurren dentro de un episodio de respiración periódica deben también ser registradas como apneas individuales". Las apneas centrales aisladas se discriminaron de las asociadas a respiración periódica mediante el análisis individual de cada estudio.

Para determinar si existía alguna correlación entre la $\mathrm{SpO}_{2}$ y la RP, se determinó la relación entre el tiempo en minutos, con $\mathrm{SpO}_{2} \geq 91 \%$, y el tiempo en minutos con $\mathrm{SpO}_{2}<91 \%$. Este coeficiente fue denominado coeficiente de saturación en el sueño (CSS) y se utilizó para hacer la correlación, que fue evaluada mediante la prueba de Spearman.

\section{RESULTADOS}

Un total de 35 niños cumplieron con los criterios de inclusión. Las polisomnografías se realizaron entre octubre de 2012 y mayo de 2013. El grupo estuvo conformado por 18 niños y 17 niñas. De ellos, 9 estuvieron entre los 30 y los 59 días de edad; 13, entre los 60 y los 89 días; y 13, entre los 90 y los 120 días. No se encontraron diferencias significativas para los grupos de edad para la $\mathrm{SpO}_{2}$, la RP o el IAC. Estos parámetros presentaron distribuciones anormales con desviación hacia la derecha (véanse los resultados principales en la Tabla 1).

La mediana para la $\mathrm{SpO}_{2}$ fue de $92 \%$ (Figura 1). La diferencia de la $\mathrm{SpO}_{2}$ entre los p5 y p25 fue del $4 \%$, cifra igual a la diferencia entre los p 25 y p95 (Figura 2). Con frecuencia, la $\mathrm{SpO}_{2}$ cayó a valores por debajo del $80 \%$; estas caídas se caracterizaron por ser cortas y de recuperación rápida.

La RP tuvo una mediana de $4,9 \%$ y fue del

Tabla 1. Parámetros respiratorios de la polisomnografía en 35 lactantes de 1 a 4 meses de edad, a $2560 \mathrm{~m}$ de altura

\begin{tabular}{|c|c|c|c|}
\hline Edad (semanas) & Promedio & 11,1 & DE 3,8 \\
\hline TTS (minutos) & Promedio & 250 & DE 32 \\
\hline Tiempo de sueño activo/REM; minutos (\%) & Promedio & $112(45 \%)$ & DE 28 \\
\hline Tiempo de sueño pasivo/NREM; minutos (\%) & Promedio & $142(55 \%)$ & DE 28 \\
\hline $\mathrm{SpO}_{2}$ en TTS & Mediana $92 \%$ & p5 86\% & p95 94,0\% \\
\hline$\% R P$ & Mediana $4,9 \%$ & p5 $0,2 \%$ & p95 46,8\% \\
\hline IACT/hora & Mediana 23,7 & p5 0,9 & p95 130,2 \\
\hline IACA/hora & Mediana 4,5 & p5 0,0 & p95 47,0 \\
\hline IACARP/hora & Mediana 15,4 & p5 0,9 & p95 105,0 \\
\hline Valor más bajo de $\mathrm{SpO}_{2}$ en TTS & Mediana 77\% & p5 61\% & p95 85\% \\
\hline CSS & Mediana 3,9 & p5 0,4 & p95 26,2 \\
\hline
\end{tabular}

TTS: tiempo total de sueño; p: percentil; RP: respiración periódica; IACT: índice total de apnea central; IACA: índice de apnea central aislada; IACARP: índice de apnea central asociada a respiración periódica; CCS: coeficiente de saturación en el sueño; $\mathrm{SpO}_{2}$ : saturación de oxígeno. 
9\% para el sueño con movimiento ocular rápido (rapid eye movement sleep; REM, por sus siglas en inglés) y del 2,3\% para el sueño pasivo (non rapid eye movement; NREM, por sus siglas en inglés), diferencia estadísticamente significativa $(p=0,0001)$. El IAC tuvo una mediana de 23,7/ hora. La mediana del IAC asociado a respiración periódica fue de 15,4/hora, mientras que la mediana para el IAC aislado fue de 4,5/hora. El tiempo promedio para las apneas centrales fue de 5,15 segundos (DE 2,0). La mediana para las

FIgURA 1. Saturación de oxígeno durante el sueño en lactantes de 1 a 4 meses edad, a 2560 m de altura en comparación con los valores a nivel del mar

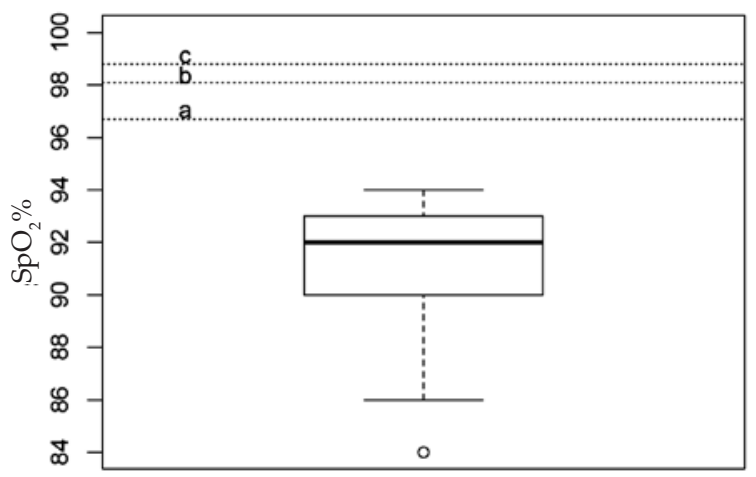

a, b, c= percentiles 5, 25 y 75 de la saturación de oxígeno durante el sueño a nivel del mar en lactantes de 1 a 4 meses de edad. ${ }^{10}$

$\mathrm{SpO}_{2}$ : saturación de oxígeno.

FIGURA 2. Valores de saturación de oxígeno por percentiles durante el sueño en niños de 1 a 4 meses, a 2560 m de altura

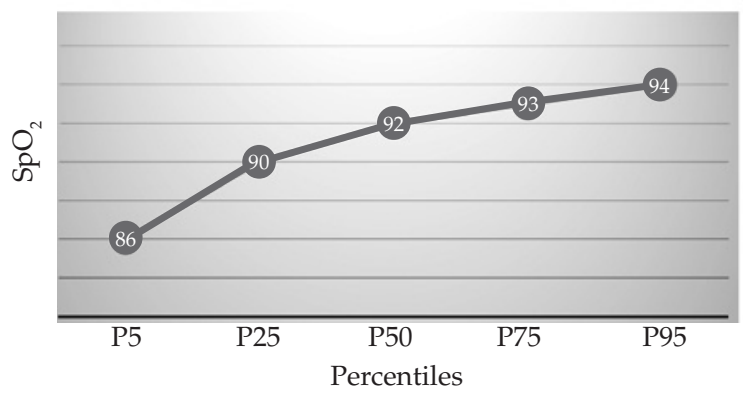

$\mathrm{SpO}_{2}$ : saturación de oxígeno hipopneas centrales fue de 0 . Los índices para las apneas obstructivas y mixtas fueron de 0 . La mediana para el CSS fue de 3,9. No encontramos correlación entre la RP y el CSS (Figura 3).

\section{DISCUSIÓN}

La $\mathrm{SpO}_{2}$ encontrada por nosotros fue claramente inferior a la reportada por Schlüter et al., a nivel del mar, quienes, en niños de 1 a 4 meses, describen una mediana de $98,1 \%$ (percentil 5, 95\% - percentil 95, 99,5\%). ${ }^{10}$ Por su parte, la RP fue significativamente mayor que la publicada por Kelly et al., a nivel del mar, quienes la encontraron por debajo del 1\% en lactantes de 2 a 4 meses $^{11}$ y por Schlüter et al. con valores por debajo del $0,5 \%$ para niños de entre 1 y 4 meses. $^{10}$ El hecho de que la RP se incrementa con la altura tiene bases fisiológicas ${ }^{12}$ y ha sido previamente reportado en lactantes. ${ }^{13}$ Nuestro hallazgo de que la RP es más alta durante el sueño REM, en comparación con el NREM, ha sido reconocido desde 1977.14

El IAC, en este estudio, es superior al reportado a nivel del mar con un valor de 5-10/ hora para lactantes de 1 a 4 meses. ${ }^{10}$ Sin embargo, al descontar las apneas centrales asociadas a respiración periódica, la mediana para el IAC, en nuestros datos, fue similar a la reportada a baja altura. Estos resultados sugieren que la discriminación entre las apneas centrales aisladas y las asociadas a la RP es crítica en este grupo de edad en la altura. Si se ignora esta diferenciación, el IAC será, en buena medida, un reflejo del porcentaje de RP. El aumento de la RP en la altura en lactantes ha sido reportado previamente por

Figura 3. Ausencia de correlación entre el coeficiente de saturación en el sueño y el porcentaje de respiración periódica en lactantes de 1 a 4 meses de edad, a $2560 \mathrm{~m}$ de altura $(r=0,057 ; p=0,278)$

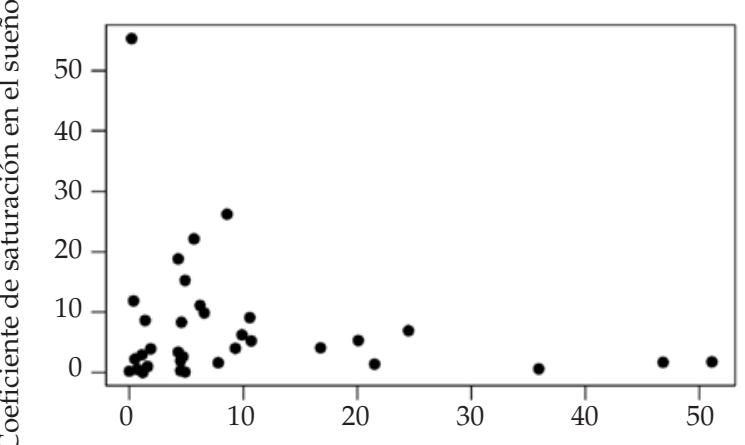

Porcentaje de saturación periódica en el sueño total 
Parkins et al. Estos autores analizaron el patrón respiratorio en 34 niños (con promedio de edad de 3,1 meses), que se expusieron, estando a nivel del mar, a oxígeno al 15\% (equivalente a una presión barométrica de $582 \mathrm{mmHg}$ ). Los resultados mostraron que las apneas asociadas a $R P$ aumentaron 3,5 veces en comparación con un incremento de apenas el 0,15 de las apneas aisladas. ${ }^{13}$

Recientemente, se publicó un estudio similar al nuestro realizado a $2640 \mathrm{~m}$ de altura. ${ }^{2}$ Los resultados coinciden en cuanto a la $\mathrm{SpO}_{2}$, pero el IAC y la RP en nuestro caso fueron mayores. Estos autores reportan un número significativo de apneas obstructivas que nosotros no encontramos.

$\mathrm{La}$ ausencia de correlación entre la $\mathrm{SpO}_{2}$ (evaluada a través del CSS) y la RP sugiere que la $\mathrm{SpO}_{2}$ menor se debe a la presión disminuida de oxígeno en la altura y no al incremento en la RP. En consecuencia, la decisión de administrar o no oxígeno debería tomarse basándose en la $\mathrm{SpO}_{2}$ y no con la intención de cambiar los valores de la RP o del IAC. Nosotros no encontramos apneas obstructivas ni mixtas. Valores de este parámetro cercanos a 0 , en este grupo de edad, han sido reportados en Alemania ${ }^{10}$ y Bélgica. ${ }^{15}$

Nuestro hallazgo relacionado con el hecho de que la diferencia de la $\mathrm{SpO}_{2}$ entre los percentiles 5 y 25 es igual al valor observado entre los percentiles 25 y 95 indica un comportamiento fisiológico diferente del $25 \%$ de los niños en comparación con el $75 \%$ restante. Podría hipotetizarse que estos lactantes tienen un mayor nivel de reactividad vascular pulmonar relacionada con la hipoxia hipobárica.

Considerando la curva de disociación de la hemoglobina, los datos obtenidos para la $\mathrm{SpO}_{2}$ pueden ser útiles como una aproximación a lo que ocurre en $\pm 300 \mathrm{~m}$ alrededor de los $2500 \mathrm{~m}$ de altura. En este rango, están ciudades como México D. F. (México) con 21 millones de habitantes, Bogotá (Colombia) con 8 millones, Addis Abeba (Etiopía) con 2,7 millones, Saná (Yemen) con 2,5 millones, Quito (Ecuador) con 2,3 millones, Arequipa (Perú) y Toluca (México) con 0,8 millones y Cochabamba (Bolivia), Quetzaltenango (Guatemala) y Asmara (Eritrea) con 0,6 millones.

\section{CONCLUSIÓN}

$\mathrm{La} \mathrm{SpO}_{2}$ fue inferior a los valores del nivel del mar, y la RP y el IAC, mayores. Cuando las apneas asociadas a RP se descontaron, el IAC fue similar al del nivel del mar.

\section{Agradecimientos}

A los padres de los niños que hicieron posible el estudio. A Sandra Rocío Morales por su trabajo en el entrenamiento de las técnicas en Cuenca y a Marta Pizano y a Lewis Grenville por su ayuda en la traducción de este artículo al inglés.

\section{REFERENCIAS}

1. Torres $\mathrm{Y}$, Osorio L, Ramos N. Medición de los valores de oximetría de pulso durante sueño, vigilia y succión en neonatos sanos en Bogotá (2640 metros de altura sobre el nivel del mar). Avances Pediátricos 1999;1:2-8.

2. Duenas-Meza E, Bazurto MA, Gozal D, González-García $\mathrm{M}$, et al. Overnight polysomnographic characteristics and oxygen saturation of healthy infants, 1 to 18 months of age, born and residing at high altitude (2,640 meters). Chest 2015: Epub 2015 Mar 26.

3. Salas AA. Pulse oximetry values in healthy term newborns at high altitude. Ann Trop Paediatr 2008;28(4):275-8.

4. Gonzáles GF, Salirrosas A. Arterial oxygen saturation in healthy newborns delivered at term in Cerro de Pasco (4340 m) and Lima (150 m). Reprod Biol Endocrinol 2005;3:46.

5. Niermeyer S, Yang P, Shanmina, Drolkar, et al. Arterial oxygen saturation in Tibetan and Han infants born in Lhasa, Tibet. N Engl J Med 1995;333(19):1248-52.

6. Niermeyer S, Shaffer EM, Thilo E, Corbin C, et al. Arterial oxygenation and pulmonary arterial pressure in healthy neonates and infants at high altitude. J Pediatr 1993;123(5):767-72.

7. Alduncin J,Grañana N, FollettF,MusanteG, etal. Problemas respiratorios durante el sueño en lactantes nativos del altiplano argentino. Arch Argent Pediatr 2005;103(1):14-22.

8. Pérez A, Rodríguez N, Gil JF, Ramírez GA. Tamaño de la muestra: a computer program to estimate the required sample size and power in clinical research. J Clin Epidemiol 1999;52 Suppl 1:38S.

9. Berry RB, Brooks R, Gamaldo CE, Harding SM, et al. The AASM Manual for Scoring of Sleep and Associated Events: Rules, Terminology and Technical Specifications, Version 2.0. Darien, IL: American Academy of Sleep Medicine; 2012.

10. Schlüter B, Buschatz D, Trowitzsch E. Perzentilkurven polysomnographischer parameter für das erste und zweite Lebensjahr. Somnologie (Berl) 2001;5(1):3-16.

11. Kelly DH, Stellwagen LM, Kaitz E, Shannon DC. Apnea and periodic breathing in normal full-term infants during the first twelve months. Pediatric Pulmonol 1985;1(4):215-9.

12. Whitelaw W. Mechanisms of sleep apnea at altitude. Adv Exp Med Biol 2006;588:57-63.

13. Parkins KJ, Poets CF, O’Brien LM, Stebbens VA, et al. Effect of exposure to $15 \%$ oxygen on breathing patterns and oxygen saturation in infants: interventional study. BMJ 1998;316(7135):887-91.

14. Hoppenbrouwers T, Hodgman JE, Harper RM, Hofmann E, et al. Polygraphic studies of normal infants during the first six months of life: III. Incidence of apnea and periodic breathing. Pediatrics 1977;60(4):418-25.

15. Kato I, Franco P, Groswasser J, Kelmanson I, etal. Frequency of obstructive and mixed sleep apnea in 1,023 infants. Sleep 2000;23(4):487-92. 